\title{
Understanding the Basics of Aviation Noise
}

\author{
Denis Gély and Ferenc Márki
}

\begin{abstract}
This chapter deals with the description of the physical mechanisms of noise, the noise perception and the annoyance induced by air traffic in the aeronautics domain. The authors introduce the basics of aviation noise, describe the main characteristics of the noise emitted by an aircraft in flight, recall the fundamental laws of the audition and noise perception and present the specific context for the annoyance due to aviation noise. This chapter presents and details, as simply as possible, the complex relationships between physical phenomena and noise perception in order to highlight the key notions in aviation noise issues. The readers will find answers to many usual and legitimate questions, for example what is the relationship between the perceived noise and the level of the physical noise related to the European ACARE goals which are expressed sometimes as EPNL reduction in $\mathrm{dB}$ or sometimes as perceived noise reduction in percent.
\end{abstract}

Keywords Physical noise $\cdot$ Source directivity $\cdot$ Annoyance $\cdot$ Noise perception $\cdot$ Audition $\cdot$ Metrics $\cdot$ Loudness $\cdot \mathrm{EPNdB} \cdot$ Community noise $\cdot$ Noise contour mapping $\cdot$ ACARE goals $\cdot$ ICAO

\section{Context of Aviation Noise}

Over the last decades and since the' $60 \mathrm{~s}$, civil aircraft noise has been mitigated significantly: the acoustical energy emitted by average modern civil aircraft-directly related to the noise it is responsible for-is somehow only $10 \%$ of an aircraft of the same size in the' $60 \mathrm{~s}$. This success was made possible thanks to specific research efforts which focused on reducing the aircraft noise at its source.

Physical Mechanisms, Metrics and Perception

D. Gély $(\bowtie)$

ONERA, The French Aerospace Lab, Châtillon, France

e-mail: denis.gely@onera.fr

F. Márki

Budapest University of Technology and Economics, Budapest, Hungary

e-mail:marki@vik.bme.hu 
Twenty years ago, at the beginning of the twenty-first century, the International Civil Aviation Organisation (ICAO) promoted the "balanced approach" for more stringent international regulations on air traffic noise. In Europe, the Advisory Council for Aeronautics Research in Europe (ACARE) targeted a further 50\% reduction by 2020 and $65 \%$ by 2050 of the perceived noise, with respect to the year 2000 as reference.

To understand the basics of aviation noise and its challenges, this chapter aims to describe simply and in short the key knowledge related to it.

Acoustics is a scientific discipline, which combines the physical aspects for describing mechanical phenomena and the physiological aspects for characterising the auditory sensation. Therefore, physical mechanics and neurosciences are inseparable for understanding and interpreting the auditory perception induced by noise and, finally, for evaluating the annoyance. To deal with this last aspect, which goes beyond physics, it is necessary to understand other sciences, human, psychological and sociological.

Many noise sources surround us, like the manufacturing industry, automotive, railway, and, of course, aviation; they all generate annoyance. Air traffic activity is subject to the paradoxical situation: for equivalent noise levels, aviation noise is perceived as the most annoying than railway noise or road traffic noise (Fig. 1).

This comparison illustrates the importance of perception, possibly including the non-acoustics factors (see Chap. 10), and help explain why the ACARE goals for noise were targeted as a mitigation of perceived noise:

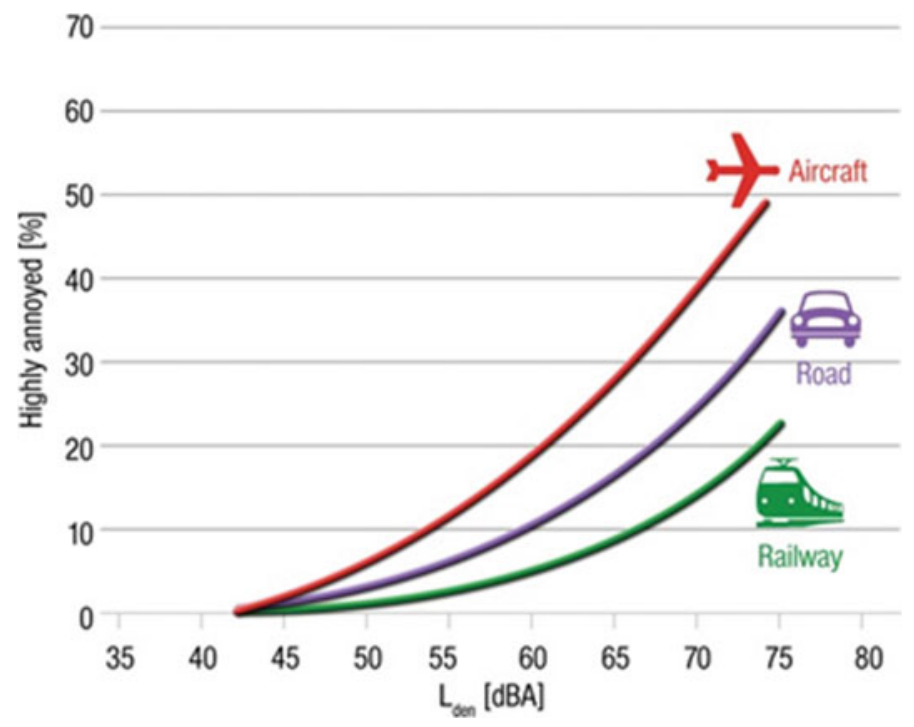

Fig. 1 Comparison of annoyance due to transportation systems. Credit European Heart Journal 2014) 
$>50 \%$ reduction of perceived noise at horizon 2020, equivalent to $10 \mathrm{EPNdB}$ reduction, related to the year 2000

$>65 \%$ reduction of perceived noise at horizon 2050, equivalent to $15 \mathrm{EPNdB}$ reduction, related to the year 2000

In the following paragraphs, we will go more into details and explain what the terms perceived noise or $E P N d B$ mean.

So the key question, related to community noise due to air traffic is:

What is the relationship between the perceived noise and the level of the physical noise?

\section{Basics of Physical Mechanisms, Metrics and Perception}

\section{Sound Pressure Level and Loudness}

The main physical quantities of acoustics are sound pressure, -power and -intensity. The physical units of these quantities, in the International System, are respectively Pascal (Pa), Watt (W) and Watt per square meter (W/ $\mathrm{m} 2)$. The acoustic power of a small drone is about $10^{-6} \mathrm{~W}$, while the one of a jet-aircraft is about $10^{6} \mathrm{~W}$. Such a large range of values to consider brings the acoustician to express the physical quantities using the Logarithm function and a value which serves as a reference because this approach compresses the scales into a meaningful interval.

For the sound pressure specifically, the Sound Pressure Level (SPL) in decibels replaces the pure physical quantity "sound pressure" and is defined as:

$$
20 * \log _{10} \frac{p}{p_{0}}[d B]
$$

where $p$ represents the instantaneous pressure, and $p_{0}$ the reference level of $2 * 10^{-5} \mathrm{~Pa}$.

By convention, the reference pressure was taken equal to $2 * 10^{-5}$ Pascals, because this corresponds to the threshold of average human ear audibility at $1000 \mathrm{~Hz}$. It is interesting to point out that the sound pressure level in decibel is referenced thus to a physiological characteristic. By using this, a sound having a level just "around" the audibility thresholds would result in $0 \mathrm{~dB}$ (SPL). Moreover, audition tests have shown that the perception of sound, i.e. the subjective feeling of sound strength (called loudness in everyday life) varies with the logarithm of the excitation, so that's one more reason to use it. Finally, decibel was chosenbecause one decibel corresponds to the human discrimination threshold in level, i.e. the just audible amount of change of sound intensity.

If we have to compute the sum of several (independent) noise sources (e.g. $L_{1}$ and $L_{2}$ ), we have to compute the resulting sound level $\left(L_{\text {sum }}\right)$ by adding their (sound) intensities, i.e. with the following equation: 


$$
L_{\text {sum }}=10 * \log _{10}\left(10^{L_{1} / 10}+10^{L_{2} / 10}\right) .
$$

This starts to be a bit too mathematical, but exactly because of this, the following very interesting facts result, worth to note:

$$
80 \mathrm{~dB}_{\mathrm{SPL}}+80 \mathrm{~dB}_{\mathrm{SPL}}=83 \mathrm{~dB}_{\mathrm{SPL}}
$$

Or even

$$
0 \mathrm{~dB}_{\mathrm{SPL}}+0 \mathrm{~dB}_{\mathrm{SPL}}=3 \mathrm{~dB}_{\mathrm{SPL}}
$$

It can be shown that by adding two levels with a significant difference, e.g. of $15 \mathrm{~dB}$, the result is roughly equal to the greater level:

$$
80 \mathrm{~dB}_{\mathrm{SPL}}+65 \mathrm{~dB}_{\mathrm{SPL}}=80 \mathrm{~dB}_{\mathrm{SPL}}
$$

Unfortunately, as you will shortly see, sound reduction sensation does not follow this rule. So when we talk about noise reduction, we always have to specify what we reduce: sound pressure level, sound intensity or the perceived loudness.

The audition characteristics have been studied at the beginning of the twentieth century. In 1933, Fletcher \& Munson, engineers at Bell labs, showed that human perception presents a double non-linearity in frequency (i.e. how low or high pitched the sound is) and in level [1]. Figure 2 shows the equal loudness level contours for pure sinus tones, which have been internationally standardised, and were obtained by statistical tests over a large group of young people with normal hearing. Each of the curves represents the necessary SPL of a pure tone at a given frequency to be perceived equally loud as other frequencies on the same curve. The curves are denoted with the metric phon, and their value corresponds to the SPL of the $1 \mathrm{kHz}$ tone. (See labelling of the curves at the $1000 \mathrm{~Hz}$ vertical line.)

Approaching these curves, from the other side, for differently pitched tones but with the same SPL, generally, the (perceived) loudness falls off at low and at high frequencies (below $500 \mathrm{~Hz}$ and above $4 \mathrm{kHz}$ ). Please note that the curves are not the same at different levels, although they seem to be quite similar. This means that the "frequency-weighting" nature of our hearing depends on how loud the sound actually is. Let us simply remember that our loudness sensation is very non-linear in relation to both frequency and absolute sound pressure level.

When acousticians have to measure sound events (with the goal to express objectively what people would say about the loudness of the event), they apply-most of the time-the so-called A-weighting. This takes into account the characteristics of the ear, and therefore makes the measuring instruments "listen" more like humans by weighting low and high frequencies to follow approximately (inversely) the equal loudness curve of 40 phons. The A-weighted sound level has been shown to correlate extremely well with a subjective response and is therefore widely used. This is the reason why you can often see $\mathrm{dB}(\mathrm{A})$ instead of $\mathrm{dB}$. 


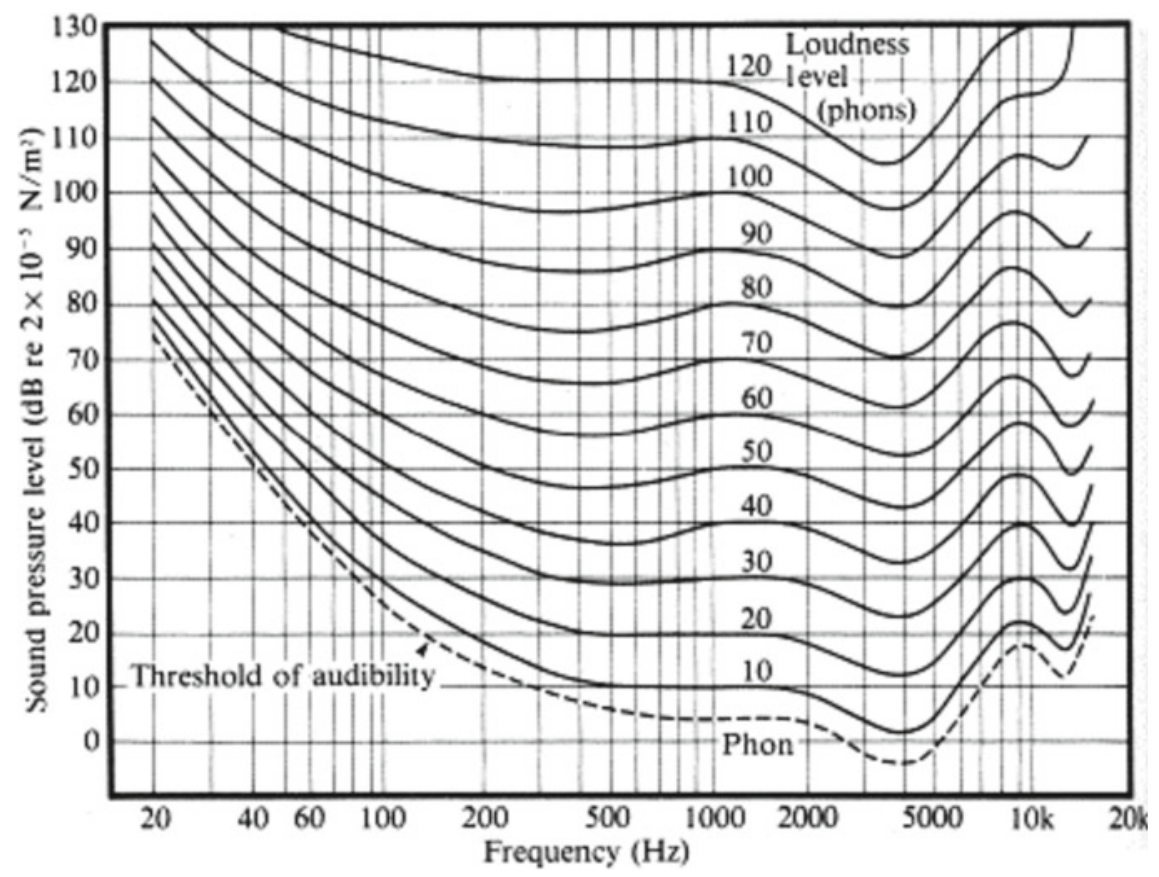

Fig. 2 Standardised equal loudness contours for pure tones

\section{Temporal Behaviour: Peak/instantaneous Levels versus Equivalent Levels}

An interesting effect of human hearing is called masking. This describes the scenario when a quieter sound cannot be heard in the presence of a louder one. On earth practically, but in cities without any doubt, there is never total silence. Sound sources can be natural, such as dogs barking, birds singing, wind, rain or even human voices, etc., but can also be artificially generated like traffic noise, industrial or construction noise, machine noise from gardening, music, etc. Many of these sounds form together a more or less steady, so-called background noise. So when a specific sound event happens, it is either low enough to vanish in the background noise (as this latter masks the quieter sound), or it is high enough to be heard. Our brain is used to living in a noisy world and tries to suppress background noise, i.e. make it unperceived for us (however, this activity certainly causes fatigue...). The more a sound event emerges from the background, the more it attracts our attention. When the sound is welcomed, then we are happy (a nice bird, good music, etc.). If, on the other hand, it is unpleasant, then it causes annoyance. Naturally, high background noise can also cause annoyance, but a disliked sound emerging from the background noise is often more annoying because it cannot be suppressed by the brain. In the long term, if we hear the same sound several times, our brain learns it, and tries to suppress it too, 
but whether this succeeds depends highly on (1) how strongly it emerges from the background, (2) how fast it becomes audible (slowly or suddenly), (3) how long it lasts and (4) whether it is constant for a while or its loudness fluctuates.

Unfortunately, aircraft noise is hard to suppress, as it often reaches a significantly louder maximum loudness than the background noise (point 1), it lasts for a too-short time to get used to it (point 2), it is by far not constant (point 4), but at least it is not fast increasing/decreasing its level (point 3). The most important negative aspect of this is how high the noise level is. In acoustics, this is called the maximum sound level and is denoted by $L_{\text {max }}$. This is completely different from the average sound level, which is called in the literature equivalent sound level and is denoted by $L_{e q}$. (If the measurements were performed with A-weighting then $L_{A, \max }$ and $L_{A, e q}$ respectively, or in its simplest text form: LAeq and LAmax.) The use of $L_{e q}$ is reasonable for more or less constant noise sources, like traffic noise, and it is beneficial, as it expresses the overall exposure to noise into just one number. Unfortunately, this became the quasi-standard to measure the effect of noise and thus, it is also used for aircraft noise. As you will see in later chapters, this selection is far from optimal, but to defend it, there is no obviously better metric to replace it. Recent research reveals, for example, that the maximum level is one important metric to be used alongside the average levels because above a certain level, it cannot distract us from what we are doing during the day, while currently, it wakes us up during the night.

\section{Lden: Day Evening Night Level}

The $L_{d e n}$ (Day Evening Night Sound Level) is the average sound level over a $24 \mathrm{~h}$ period. To take into account, the annoyance induced by the noise during the sleeping and the rest periods, a penalty of $5 \mathrm{~dB}$ is added for the evening period (usually from 19:00 to 23:00) and $10 \mathrm{~dB}$ for the night period (usually from 23:00 to 7:00). The Lden is used for noise contour mapping around an airport allowing the identification of critical areas in terms of community noise (i.e. areas subjected to noise regulations, see Fig. 3).

According to a European Environmental Report, almost 3 million people in Europe are exposed to air traffic noise above $55 \mathrm{~dB}$ Lden, which is the EU threshold for excess exposure defined in the Environmental Noise Directive [https://www.eea.europa. eu/airs/2018/environment-and-health/environmental-noise]. For comparison, 125 million people are affected by noise levels from road traffic greater than $55 \mathrm{~dB}$ Lden, including more than 37 million exposed to noise levels above $65 \mathrm{~dB}$ Lden! [European Environment Agency, www.eea.europa.eu/publications/managing-exposureto-noise-in-europe]. 


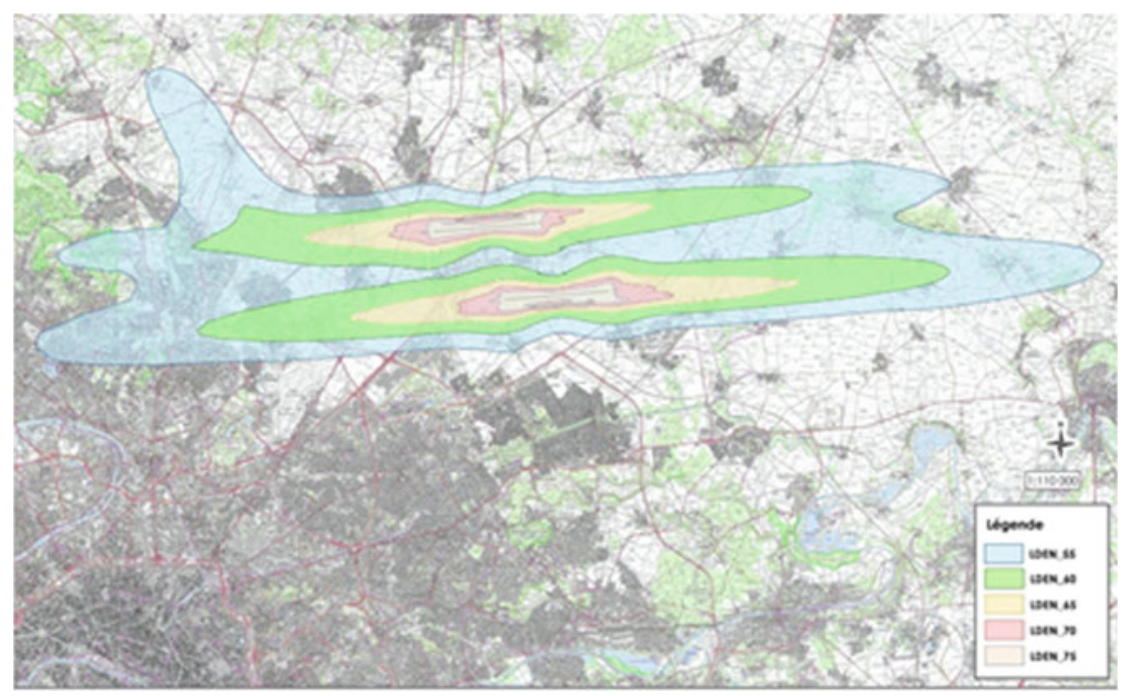

Fig. 3 Lden contours map around Roissy-CDG Airport (France)

\section{Source Directivity}

In everyday life, we are used to teaching our children to turn towards us, when they talk to us. We do this because most sound sources have uneven directivity. Directivity expresses how much sound is radiated into one or the other direction. If this wouldn't make our life complicated enough to describe sound sources, we face the fact that directivity is most of the time also frequency-dependent. This means that for example, a source radiates the lower-pitched "parts" of the sound evenly into every direction (behaving as omnidirectional), while the higher-pitched content of the sound is radiated strongly into one direction (behaving as directional). So we hear low- and high-tuned sound components quieter than mid-tuned ones. This means, in practice, if a directional source is directed towards us, we definitely hear it louder than a source that is turned away from us. And this could happen also with aircraft noise. Noise radiated from aircraft engines is strongly directionally, specifically the direction along the axis of their engines. When the aircraft takes off, the engines exhaust point towards the ground, causing higher sound levels there, generally, but these are also perceived to be as being even louder because of the higher mid-tone content. Additionally, when a taking-off aircraft turns, it rapidly draws a "trace" on the ground with strongly directed sound. For people living there, this means that the sound becomes louder more quickly, and it also reaches a higher maximum sound level than for people who are not affected by the directed part of the engine noise. So let us keep in mind that the directional behaviour of engine noise also causes a measurable sound level difference at some locations, and the perceived noise is even higher. However, related to the overall averaging time (i.e. a year), this more 
inconvenient fraction of time is too short to appear in numbers. This results in more annoyance without significantly increased long-term noise metrics.

Such a feature must be underlined: annoyance cannot always be described by metrics and especially by time-average metrics.

\section{Effective Perceived Noise Level: EPNL}

In aeronautics, the usual metric is the Effective Perceived Noise Level (with units EPNdB), which takes into account the duration and the tonality of a flyover of an aircraft. EPNL is calculated (see Fig. 4) from the sound recording by identifying two instants $t 1$ and $t 2$ corresponding to the instants for which the sound level is $10 \mathrm{~dB}$ lower than the maximum level (PNL max in $\mathrm{dB}(\mathrm{A})$ ). The equivalent continuous level is calculated for the period between these two instants. If there are tonal components in the sound, then a penalty is added. The equivalent continuous level is then normalised to a period of $10 \mathrm{~s}$ to obtain the EPNdB. Note that the duration and tonality of the sound add only a certain amount of decibels to the regular measurements, but overall, EPNL still remains a kind of equivalent level, a "dB" metric. Keeping the characteristics of a flyover but reducing it by a few decibels results in the same reduction in EPNdB.

Generally, we also can consider that $10 \mathrm{~dB}$ noise reduction can be achieved by the same amount of EPNdB reduction.

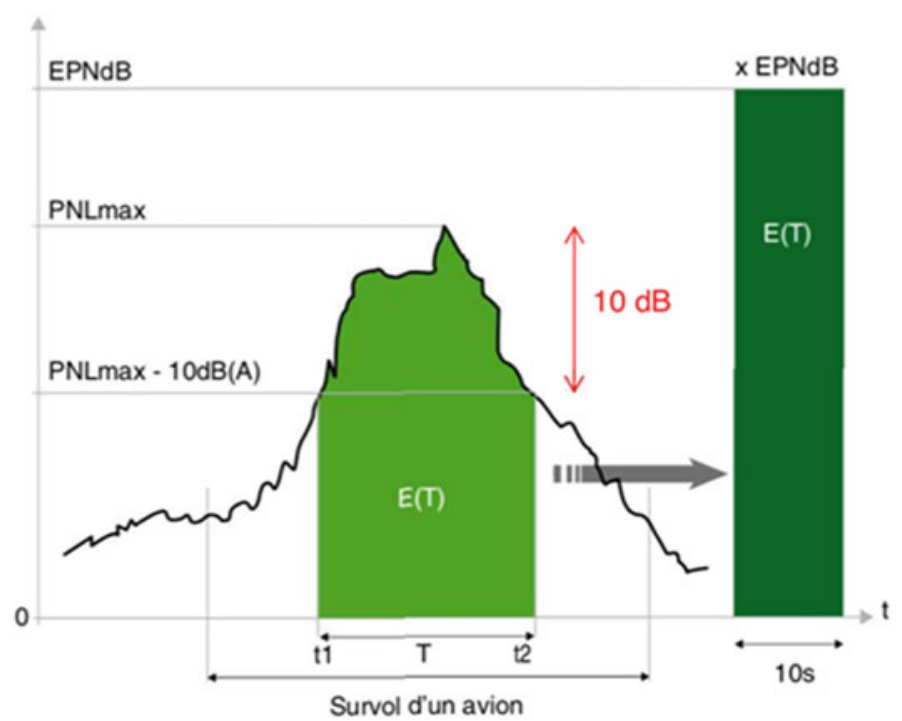

Fig. 4 EPNdB calculation process 
Now, to better understand ACARE goals, we have shortly to come back to psychoacoustics and become acquainted with a scale proportional to perceived loudness, the Sone scale. The relationship between loudness in Sones (perception) and loudness level in Phons (physics) is given by:

$$
L_{\text {sone }}=2^{\frac{L_{p h o n}-40}{10}}
$$

where $L_{\text {sone }}$ and $L_{\text {phon }}$ denote the sound level in sone vs phon respectively.

According to this formula, to half the perceived loudness $\left(L_{\text {sone }}\right)$, it must be reduced by 10 phons. Generally, for changes in sound level, we can neglect the slight deviance of equal loudness curves, and thus say that to half the perceived loudness, a level reduction of $10 \mathrm{~dB}$ is necessary.

If we apply this knowledge, we can better understand the noise reduction objectives set by ACARE in:

\begin{tabular}{l|l|l|l}
\hline ACARE goal's year & $\begin{array}{l}\text { Noise reduction in } \\
\text { EPNdB per operations }\end{array}$ & $\begin{array}{l}\text { Perceived noise }\left(\mathrm{L}_{\text {sone }}\right) \\
\text { divided by }\end{array}$ & $\begin{array}{l}\text { Perceived noise } \\
\text { reduced by } \%\end{array}$ \\
\hline 2020 & 10 & 2 & $50 \%$ \\
\hline 2050 & 15 & 2,8 & $65 \%$ \\
\hline
\end{tabular}

\section{Reference}

1. Fletcher H, Munson WA (1933) Loudness, its definition, measurement and calculation. J Acoust Soc America 5:82-108

Open Access This chapter is licensed under the terms of the Creative Commons Attribution 4.0 International License (http://creativecommons.org/licenses/by/4.0/), which permits use, sharing, adaptation, distribution and reproduction in any medium or format, as long as you give appropriate credit to the original author(s) and the source, provide a link to the Creative Commons license and indicate if changes were made.

The images or other third party material in this chapter are included in the chapter's Creative Commons license, unless indicated otherwise in a credit line to the material. If material is not included in the chapter's Creative Commons license and your intended use is not permitted by statutory regulation or exceeds the permitted use, you will need to obtain permission directly from the copyright holder.

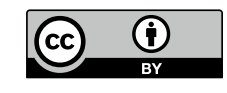

